\title{
Breakdown of the equal area law for holographic entanglement entropy
}

\author{
Fiona McCarthy, ${ }^{a, b}$ David Kubizňák ${ }^{a, b}$ and Robert B. Mann ${ }^{b, a}$ \\ ${ }^{a}$ Perimeter Institute for Theoretical Physics, \\ 31 Caroline Street North, Waterloo, ON, N2L 2Y5, Canada \\ ${ }^{b}$ Department of Physics and Astronomy, University of Waterloo, \\ Waterloo, Ontario, N2L 3G1, Canada \\ E-mail: fmccarthy@perimeterinstitute.ca, \\ dkubiznak@perimeterinstitute.ca, rbmann@uwaterloo.ca
}

ABSTRACT: We investigate a holographic version of Maxwell's equal area law analogous to that for the phase transition in the black hole temperature/black hole entropy plane of a charged AdS black hole. We consider proposed area laws for both the black hole temperature/holographic entanglement entropy plane and the black hole temperature/2point correlation function plane. Despite recent claims to the contrary, we demonstrate numerically that neither proposal is valid. We argue that there is no physical reason to expect such a construction in these planes.

KEYwORDS: AdS-CFT Correspondence, Black Holes

ARXIV EPRINT: 1708.07982 


\section{Contents}

1 Introduction $\quad 1$

2 Phase structure of charged AdS black holes 2

3 Testing holographic equal area laws 5

3.1 Entanglement entropy 5

$\begin{array}{lll}3.2 & \text { Two-point correlation function } & 8\end{array}$

$\begin{array}{llr}4 & \text { Failure of the equal area construction } & 9\end{array}$

5 The "approximate" equal area law near criticality 13

6 Conclusions 14

$\begin{array}{ll}\text { A The approximate equal area law: general argument } & 15\end{array}$

\section{Introduction}

Maxwell's equal area law, which states that two phases coexist when the areas above and below a line of constant pressure $P$ drawn through a pressure/volume curve are equal, is one of the hallmarks of thermodynamics. It provides a straightforward computational method for obtaining the coexistence boundary between any two phases (separated by a first order phase transition), and generalizes straightforwardly to any pair of conjugate thermodynamic variables. In recent years it has found utility in the thermodynamics of AdS black holes [1-3], where the magnitude of the cosmological constant is interpreted as thermodynamic pressure and the conjugate volume $V$ is obtained by differentiating the black hole mass with respect to pressure [4].

Recently there has been interest in defining equal area laws for holographic entanglement entropy [5-8] as well as two-point correlation functions [9-18]. These equal area laws have been studied for spacetimes dual to AdS black holes with phase transitions obeying an equal area law in the black hole temperature $(T)$ /black hole entropy $(S)$ plane such as the charged AdS black hole undergoing a first-order phase transition [19-21]. The holographic equal area laws have been considered in both the $T$ /entanglement entropy plane and the $T /$ geodesic length plane, as the two-point correlation function is given by the exponential of geodesic length [22]. It has been claimed in a number of cases [5, 6, 8-18] that isocharges in these planes obey Maxwell's equal area construction at the phase transition temperature of the black hole $T_{*}$, such that the areas bounded above and below the isocharge and the isotherm $T=T_{*}$ are equal, just as is true for black hole temperature and entropy. 
The similarity between holographic entanglement entropy (HEE) and black hole entropy [23] motivated the idea of an equal area law for holographic entanglement entropy, where it was first claimed [5] that there is numerical evidence for an HEE equal area law for the (near critically) charged AdS black hole in $3+1$ dimensions. It was further claimed that this equal area law for HEE sharpened the similarity between black hole entropy and HEE. However, numerical evidence that the equal area law for HEE breaks down was subsequently presented [7], the discrepancy growing as isocharges are chosen further away from their originally considered [5] near-critical values. More recently, claims that a holographic equal area law holds in the $T$ /geodesic length plane have appeared [9-18].

Here we present the results of an investigation into both proposals for a holographic equal area law. We find that any claim of an equal area law holding in either the $T /$ entanglement entropy or $T /$ geodesic length plane is untrue and unfounded. We find numerically that such equal area laws are not satisfied in either case, and explain how such erroneous claims could arise. Furthermore, we point out that there is no reason to expect this based on an appropriate consideration of the relevant thermodynamics.

\section{Phase structure of charged AdS black holes}

It is well known [19-21] that in a canonical (fixed charge) ensemble the thermodynamics of charged AdS black holes features a first order (small black hole/large black hole) phase transition, with the corresponding thermodynamics governed by the black hole free energy. Alternatively, one can describe such a phenomenon using the Maxwell equal area construction in the $T-S$ (and/or $P-V$ ) planes. Since the charged AdS black hole will serve as a testground for our investigation of validity of the holographic equal area laws, let us start by briefly recapitulating these bulk results.

A $d$-dimensional charged AdS black hole is a solution to the Einstein-Maxwell anti de Sitter action [19]

$$
I=-\frac{1}{16 \pi G} \int d^{d} x \sqrt{-g}\left[R-F^{2}+\frac{(d-1)(d-2))}{l^{2}}\right],
$$

where $l$ is the AdS length scale, given by the following metric:

$$
\begin{aligned}
& d s^{2}=-f(r) d t^{2}+\frac{d r^{2}}{f(r)}+r^{2} d \Omega_{(d-2)}^{2}, \\
& f(r)=1-\frac{m}{r^{d-3}}+\frac{q^{2}}{r^{2(d-3)}}+\frac{r^{2}}{l^{2}} .
\end{aligned}
$$

The parameters $m$ and $q$ are related to the ADM mass and charge of the black hole $M$ and $Q$ via

$$
\begin{aligned}
& M=\frac{(d-2) \omega_{(d-2)}}{16 \pi G}, \\
& Q=\frac{\sqrt{2(d-2)(d-3)} \omega_{(d-2)}}{8 \pi G} q,
\end{aligned}
$$


where $\omega_{(d-2)}$ is the area of the unit $(d-2)$-sphere $\omega_{(d-2)}=\frac{2 \pi \frac{d-1}{2}}{\Gamma\left(\frac{d-1}{2}\right)}$. The temperature $T=\frac{f^{\prime}\left(r_{+}\right)}{4 \pi}$ and entropy $S=\omega_{(d-2)} r_{+}^{d-2} / 4$ are straightforwardly computed. The solution for the gauge potential is

$$
A=\left(-\frac{1}{c} \frac{q}{r^{d-3}}+\Phi\right) d t
$$

where $c=\sqrt{\frac{2(d-3)}{d-2}}$, and $\Phi$ is a constant. Choosing $\Phi=\frac{1}{c} \frac{q}{r_{+}^{d-3}}$, with $r_{+}$the horizon radius of the black hole, the potential $A$ vanishes on the horizon. The above black hole quantities obey the following standard first law of black hole thermodynamics:

$$
d M=T d S+\Phi d Q .
$$

Specializing to $d=3+1$ dimensions, we can express $T$ as a function of $S$ and $Q$

$$
T(S, Q)=\frac{1}{4 \pi}\left(\frac{3}{l^{2}} \sqrt{\frac{S}{\pi}}+\sqrt{\frac{\pi}{S}}-Q^{2} \frac{\pi^{\frac{3}{2}}}{S^{\frac{3}{2}}}\right)
$$

and also obtain in the canonical ensemble

$$
F=M-T S=\frac{1}{4 l^{2}}\left(l^{2} r_{+}-r_{+}^{3}+\frac{3 Q^{2} l^{2}}{r_{+}}\right)
$$

for the free energy $F=M-T S$, which completely governs the thermodynamic behavior of the bulk black hole.

Namely, when isocharge lines are plotted in the $F-T$ plane, swallowtail behaviour characteristic of a first-order phase transition is observed for sufficiently small charges [19-21] (figure 1). The phase transition temperature $T_{*}$ occurs at a point at which the derivatives of the global minimum of $F$ become discontinuous, that is, at a point where the swallowtail intersects itself. As charge increases, the swallowtail diminishes and eventually terminates at a critical point characterized by $Q=Q_{\text {crit }}$ and $T=T_{\text {crit }}$ at which the phase transition becomes second order. For $Q>Q_{c r i t}$ the swallowtail no longer exists and only one phase of black holes is present.

The thermodynamic behaviour can alternatively be inferred by studying isocharge lines in the $T-S$ plane. Namely, when $T$ is plotted against $S$ for corresponding values of $Q$ (right figure 1), we see that the swallowtail corresponds to an oscillatory behavior in $T$, and the disappearance of the swallowtail at $Q=Q_{\text {crit }}$ corresponds to a point of inflection in $T$. In particular, the critical point quantities $Q_{c r i t}, S_{\text {crit }}, T_{\text {crit }}$ can be found by solving explicitly for the inflection point

$$
\frac{\partial T}{\partial S}=\frac{\partial^{2} T}{\partial S^{2}}=0
$$

together with (2.8), while the phase transition temperature $T_{*}$ (for $Q<Q_{\text {crit }}$ ) is determined from Maxwell's equal area construction [19]:

$$
\int_{S_{1}}^{S_{2}} T(S, Q) d S-T_{*}\left(S_{2}-S_{1}\right)=T_{*}\left(S_{3}-S_{2}\right)-\int_{S_{2}}^{S_{3}} T(S, Q) d S
$$



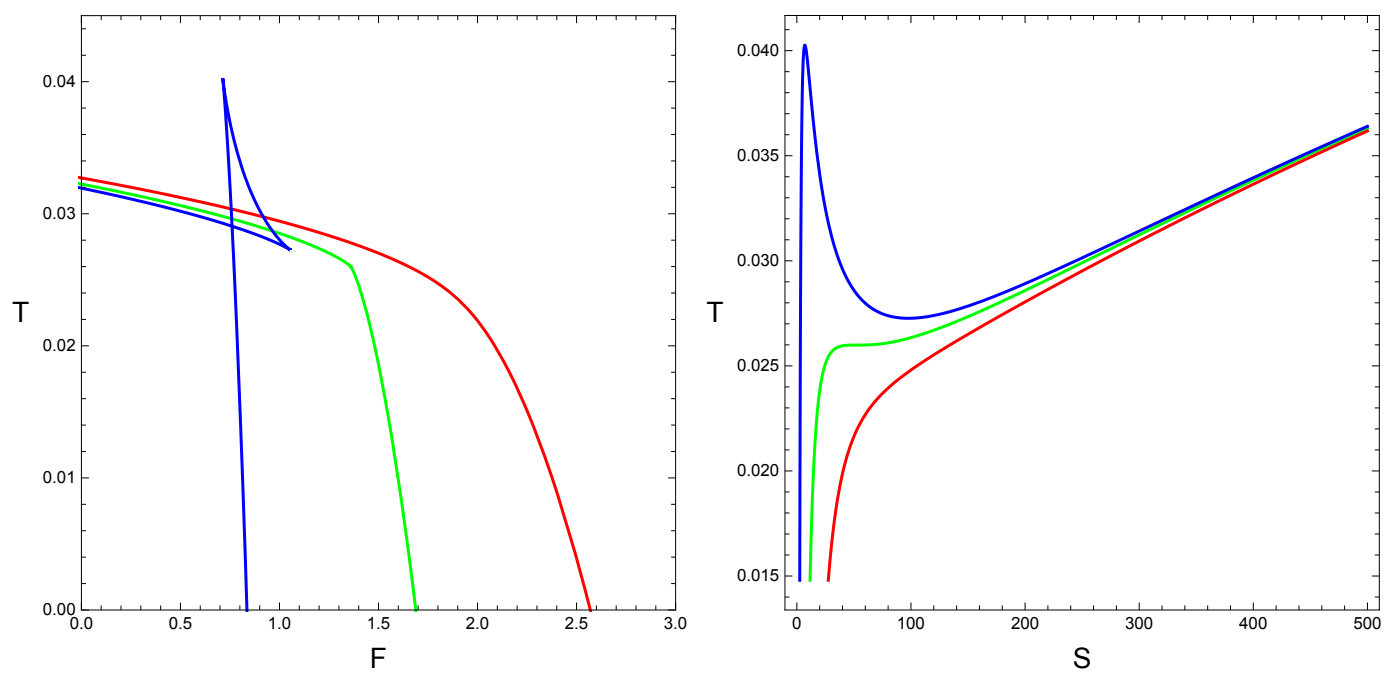

Figure 1. Phase transition of a bulk black hole. On the left, the behavior of the temperature $T$ against the free energy $F$ of a charged black hole in $d=4$ is shown. On the right is the behavior of $T$ against the entropy $S$. In each case we have plotted the critical isocharges (green), and isocharges at $Q>Q_{\text {crit }}$ (red) and $Q<Q_{\text {crit }}$ (blue). Below criticality we see the swallowtail behavior of $F$, characteristic of a first order phase transition, and the oscillatory behaviour of $T$; at criticality the swallowtail becomes a cusp in the $T-F$ plane, and an inflection point in the $T-S$ plane - the phase transition is here of second order. The AdS radius $l$ has been set to 10 for which the critical charge is $Q_{c}=10 / 6$. The values of $Q$ on these isocharges are $Q=0.5 Q_{\text {crit }}$ (blue), $Q=Q_{\text {crit }}$ (green) and $Q=1.5 Q_{\text {crit }}$ (red).

with $S_{1}, S_{2}, S_{3}$ given by the solutions of $T(S, Q)=T_{*}$ in ascending order. Graphically, this corresponds to

$$
\operatorname{Area}(\mathrm{I})=\operatorname{Area}(\mathrm{II})
$$

with Area(I) and Area(II) the areas bounded above and below by $T(S, Q)$ and $T_{*}$, as depicted in figure 2 .

It is easy to see that Maxwell's equal area law directly follows from the first law for the free energy:

$$
d F=-S d T+\Phi d Q
$$

which is a Legendre equivalent of (2.7). As $d F$ is an exact differential, we have the equal area condition:

$$
\oint S d T=0 \Rightarrow T_{*}\left(S_{3}-S_{1}\right)=\int_{S_{1}}^{S_{3}} T d S
$$

on an isocharge $(d Q=0)$, with $T_{*}$ the temperature of the phase transition isotherm, cf. eq. (2.11). Of course, the same derivation of the equal area law applies when the $T-S$ plane is (for example) replaced by the $P-V$ plane of the extended phase space thermodynamics [4]. 


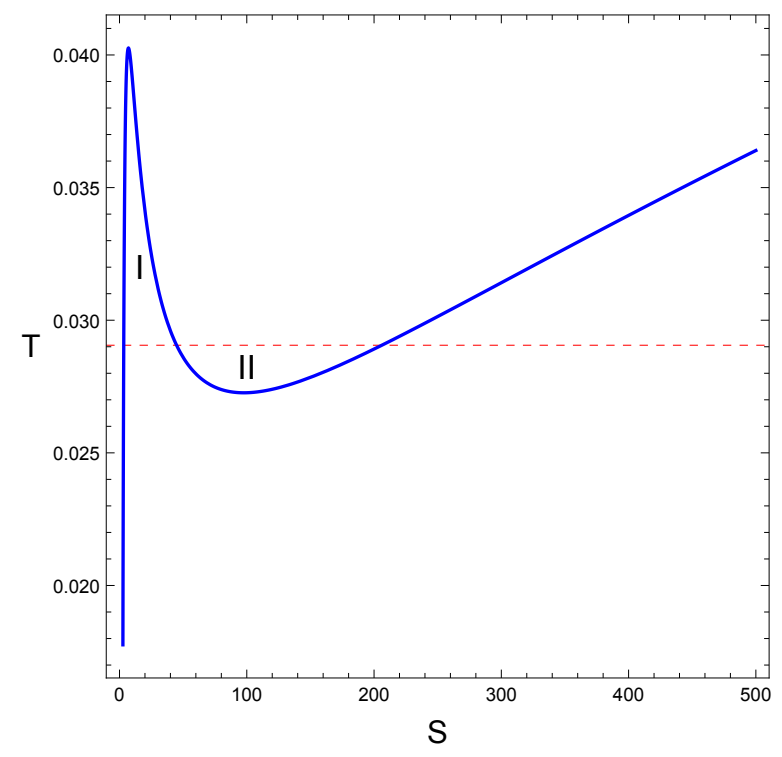

Figure 2. Maxwell's equal area law in the bulk. The phase transition temperature $T=T_{*}$ is the one at which Areas I and II bounded by the isocharge curve in the $(T, S)$ plane are equal.

\section{Testing holographic equal area laws}

The qualitatively similar behavior of HEE and black hole entropy when plotted against black hole temperature [23], cf. figure 1 and figure 3, have motivated investigations of potentially interesting phase structure in the QFT dual to a charged AdS black hole. Both entanglement entropy [5-10, 12-18] and two-point correlation functions [9-18] have been considered to this end. In both cases equal area constructions have been respectively proposed in the black hole temperature/entanglement entropy plane and the black hole temperature/two point corelation function plane, where a constant entangling region or pair of points are chosen on the boundary, and the bulk metric is varied by increasing the mass of the black hole. In this section we put both these proposals to test. Namely, we numerically investigate the behavior of holographic quantities for the CFT dual to the charged AdS black hole spacetimes. We start with the entanglement entropy.

\subsection{Entanglement entropy}

For any quantum system localized to some region $A$, the entanglement entropy is given by

$$
S_{A}=-\operatorname{Tr}_{B} \rho_{A} \log \rho_{A},
$$

where the system is partitioned into region $A$ and its complement $B$ where $\rho_{A}=\operatorname{Tr}_{B}|\psi\rangle\langle\psi|$ is the reduced density matrix describing subsystem $A$ with the system originally being in a pure state $|\psi\rangle$. A common example is that of complementary spatial volumes on a given constant time slice, their common boundary being the "entangling surface". One can express $\rho_{A}$ in the form of an effective thermal system

$$
\rho_{A}=\frac{e^{-H_{A} / T_{0}}}{\operatorname{Tr}\left(e^{-H_{A} / T_{0}}\right)}
$$


where $H_{A}$ is known as the modular Hamiltonian, and $T_{0}$ is a constant with units of temperature. Upon employing (3.1) this yields the first law

$$
T_{0} d S_{A}=\operatorname{Tr}\left(H_{A} d \rho_{A}\right) \equiv d\left\langle H_{A}\right\rangle
$$

for entanglement entropy [24, 25].

The Ryu-Takayanagi proposal [26] extends the above construction to that of a CFT in $d-1$ dimensions constructed in a spacetime corresponding to the boundary of an asymptotically bulk $\mathrm{AdS}_{d}$ spacetime (for which the quantum state of the CFT is not necessarily pure). Continuing to refer to $S_{A}$ as the entanglement entropy, their proposal states that

$$
S_{A}=\frac{A_{\Sigma}}{4 G_{d}}
$$

applied to a bulk minimal surface $\Sigma$ (with area $A_{\Sigma}$ ), whose boundary matches the entangling surface $A$ in the CFT at spatial infinity. To compute this quantity a regularization procedure is required since the minimal surface area in an asymptotically AdS bulk is formally divergent. In what follows, rather than the entanglement entropy of the excited CFT state (in the presence of a black hole), we are interested in the relative entanglement entropy

$$
S_{E}=S_{A}-S_{A}^{(0)},
$$

given by subtracting the analogous contribution $S_{A}^{(0)}$ from vacuum AdS.

Let us turn now to the calculation of the relative entanglement entropy in the charged AdS black hole spacetime. Choosing the region $A$ to be a spherical cap (as in [5]), the entangling surface can then be described by constant polar angle $\theta=\theta_{0}$, and the entanglement entropy obtained via (3.4), where the area $A_{\Sigma}$ is obtained by minimizing the action functional

$$
A_{\Sigma}=\omega_{(d-3)} \int_{0}^{\theta_{0}}(r(\theta) \sin \theta)^{d-3} \sqrt{\frac{r^{\prime}(\theta)^{2}}{f(r(\theta))}+r(\theta)^{2}} d \theta
$$

via Euler-Lagrangian variation. The relative entanglement entropy (3.5) is given by subtracting the analogous contribution from vacuum AdS. This latter contribution is explicitly known [23]:

$$
r_{0}(\theta)=l\left(\left(\frac{\cos \theta}{\cos \theta_{0}}\right)^{2}-1\right)^{-\frac{1}{2}}
$$

and the corresponding quantity $S_{A}^{(0)}$ straightforwardly computed. However the EulerLagrange system following from (3.6) must in general be solved numerically with boundary conditions

$$
\begin{aligned}
& r\left(\theta_{0}\right) \rightarrow \infty, \\
& r^{\prime}(0)=0 .
\end{aligned}
$$

where (3.8) ensures that $r(\theta)$ coincides with the entangling surface on the boundary $r \rightarrow \infty$ and (3.9) ensures regularity at the centre $(\theta=0$, the middle of the entangling surface, 

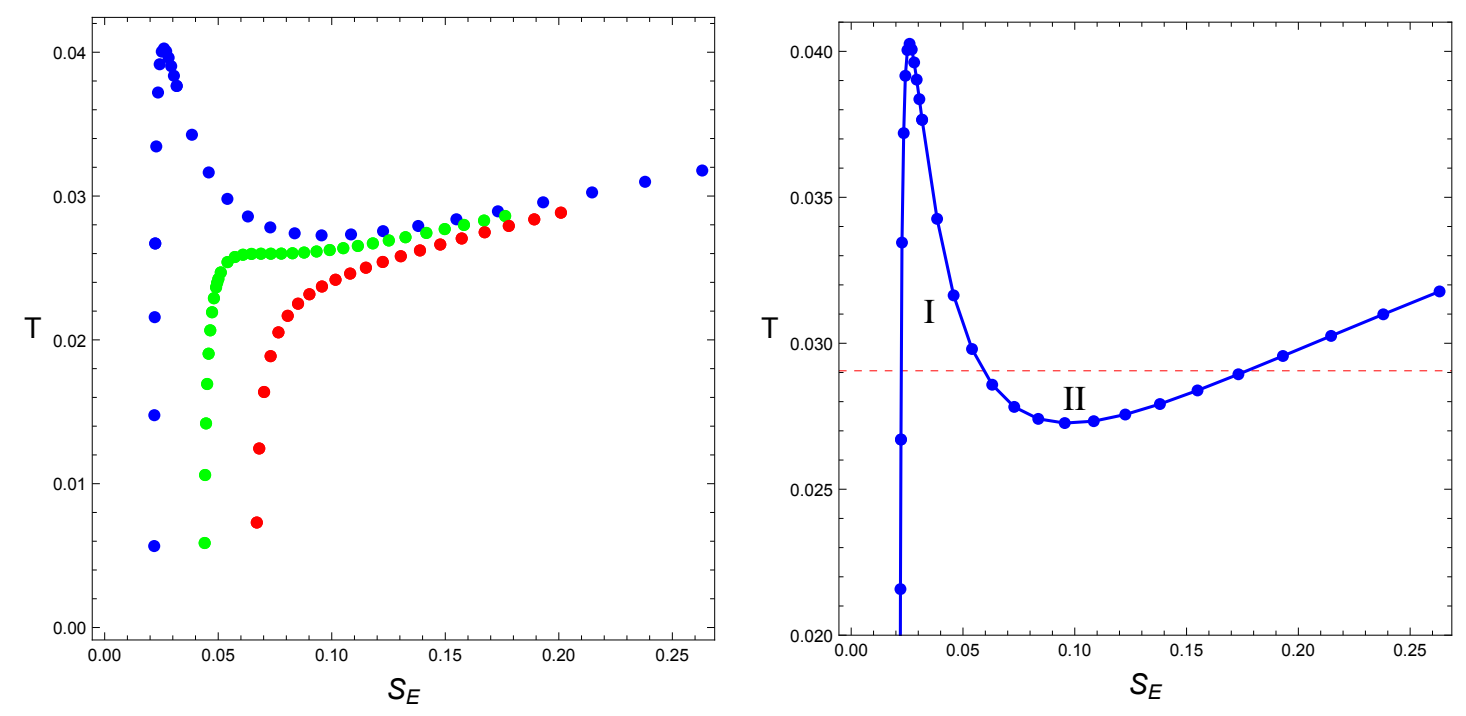

Figure 3. $T-S_{E}$ diagram for a $d=4$ charged $A d S$ black hole. On the left we see the oscillatory behavior for $Q<Q_{\text {crit }}$ (blue) and the point of inflection for $Q=Q_{\text {crit }}$ (green). On the right the Maxwell construction for entanglement entropy for $Q<Q_{\text {crit }}$ is shown; it was claimed in [5] that Areas I and II as shown are equal above and below the phase transition temperature of the black hole $T_{*}$, given by the solid line. The paramaters chosen were $l=10, \theta_{0}=0.15$, and $\theta_{c}=0.149$. Again, the values of $Q$ are $Q=0.5 Q_{\text {crit }}$ (blue), $Q=Q_{\text {crit }}$ (green) and $Q=1.5 Q_{\text {crit }}$ (red).

which is the point of maximum penetration into the bulk). Since entanglement entropy is divergent, a long-distance cut off must be introduced for regularization, which can be implemented by choosing a cut-off value $\theta_{c}<\theta_{0}$, and only integrating up to $\theta_{c}$. In our investigation we limit ourselves to considering small $\theta_{0}$.

After computing $S_{E}$ for a range of values of $T$ for a charged AdS black hole in $3+1$ dimensions, we can plot the isocharges in the $T / S_{E}$ plane; see figure 3 . Comparing figure 1 and figure 3 , which show the isocharges in the $T / S$ and $T / S_{E}$ planes respectively, we see that the behavior of black hole temperature against entanglement entropy is qualitatively similar to that against the black hole entropy. In particular, we see oscillatory behavior for charges below the critical charge $Q_{c r i t}$, a point of inflection at $Q_{c r i t}$, and monotonic increase above $Q_{c r i t}$. It is perhaps natural to consider that an equal area law holds for $Q<Q_{\text {crit }}$ (such that Areas I and II are equal on the right-side of figure 3), and indeed numerical evidence in favour of this has been presented [5].

However, as noted in [7] the apparent validity of the equal area law seems misleading, with the discrepancy growing as isocharges are chosen further away from near critical values, originally considered in [5]. To resolve this dispute, we have displayed in figure 4 the $T$ versus $S_{E}$ diagrams for various isocharges. The phase transition temperature $T_{*}$ is identified as the phase transition temperature in the bulk, where the isocharge intersects itself in the free energy diagram in figure 1. The results of the equal area law are shown in table 1 , with the relative error defined as $\frac{\operatorname{Area}(\mathrm{I})-\mathrm{Area}(\mathrm{II})}{\operatorname{Area}(\mathrm{I})} \times 100$. We see that at values of $Q$ very close to criticality, the relative error is low enough to lead one to believe an equal 


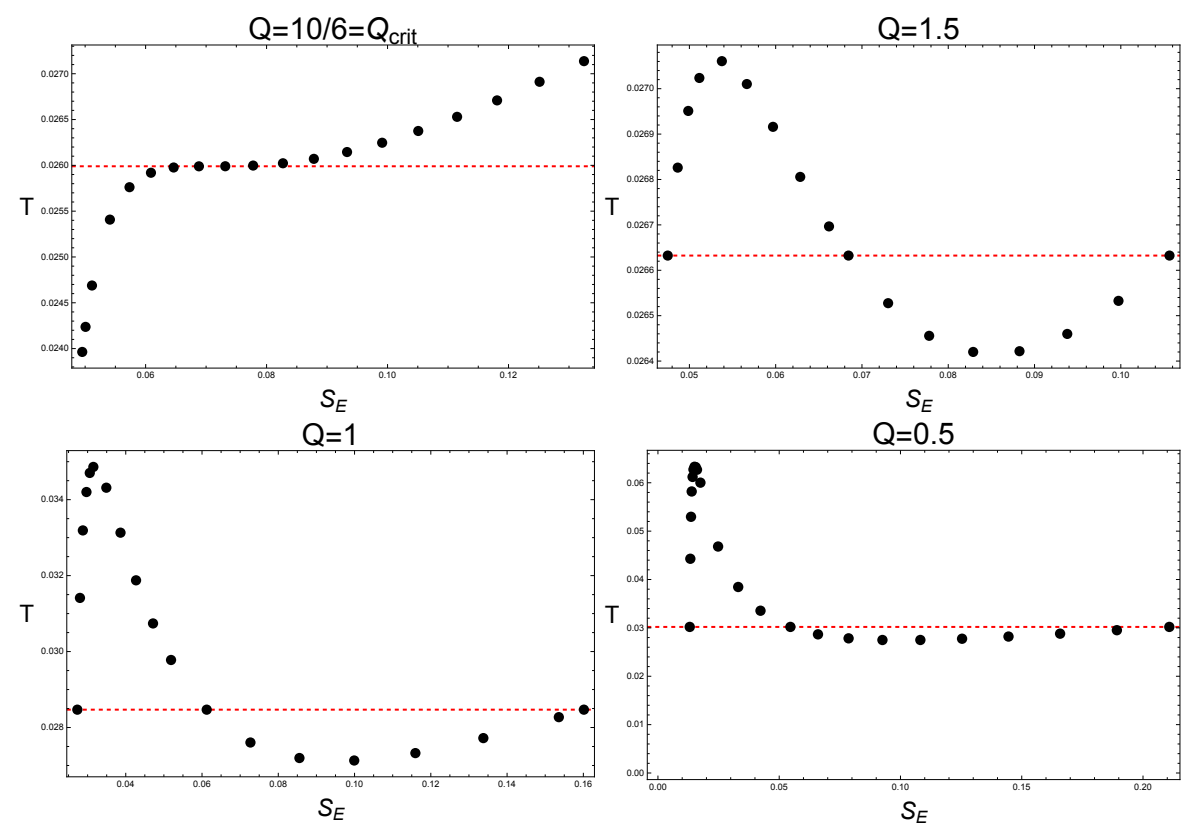

Figure 4. Charge dependence of $T-S_{E}$ diagrams. The plots are displayed for the $d=4$ charged ADS black holes, with $l=10, \theta_{0}=0.15$, and $\theta_{c}=0.149$. The phase transition temperature $T_{*}$ is plotted in red in each case.

\begin{tabular}{|c|c|c|c|c|c|}
\hline$Q$ & $Q / Q_{c}$ & $T_{*}$ & Area(I) & Area(II) & Relative error \\
\hline 1.5 & 0.9 & 0.0266324 & $5.309 \times 10^{-6}$ & $5.202 \times 10^{-6}$ & $2.02 \%$ \\
\hline 1 & 0.6 & 0.02847 & $1.040 \times 10^{-4}$ & $8.425 \times 10^{-5}$ & $19.0 \%$ \\
\hline 0.5 & 0.3 & 0.030198 & $4.745 \times 10^{-4}$ & $2.653 \times 10^{-4}$ & $44.1 \%$ \\
\hline
\end{tabular}

Table 1. Failure of the equal area law in the $T-S_{E}$ plane in $d=4$. In this table $l=10$ and $Q_{c}=5 / 3$. We see that as we move further from criticality, the relative error between Areas I and II increases and the equal area law does not hold. The plots of the results can be found in figure 4 .

area law might hold; however, as we move away from criticality, the equal area law breaks down, as noted in [7]. We checked that the equal area law also breaks down for charged AdS black holes in higher spacetime dimensions.

The disagreement between our findings and those in [5, 6, 8-18] arises both from the fact that we have probed further away from criticality than had been done, and are thus finding larger relative errors, and also from a numerical argument. As noted in [7], when the areas given by (2.14) are compared, there is less of a relative error than for the more precise areas given by (2.11). As we shall elaborate on below, such discrepancies can be huge; as much as 3 and 42 percent for $Q=0.3 Q_{c}$.

\subsection{Two-point correlation function}

We turn now to the equal-time two-point correlation function [22]

$$
\left\langle\mathcal{O}\left(t_{0}, x_{i}\right) \mathcal{O}\left(t_{0}, x_{j}\right)\right\rangle \approx e^{-\Delta L\left(x_{i}, x_{j}\right)}
$$



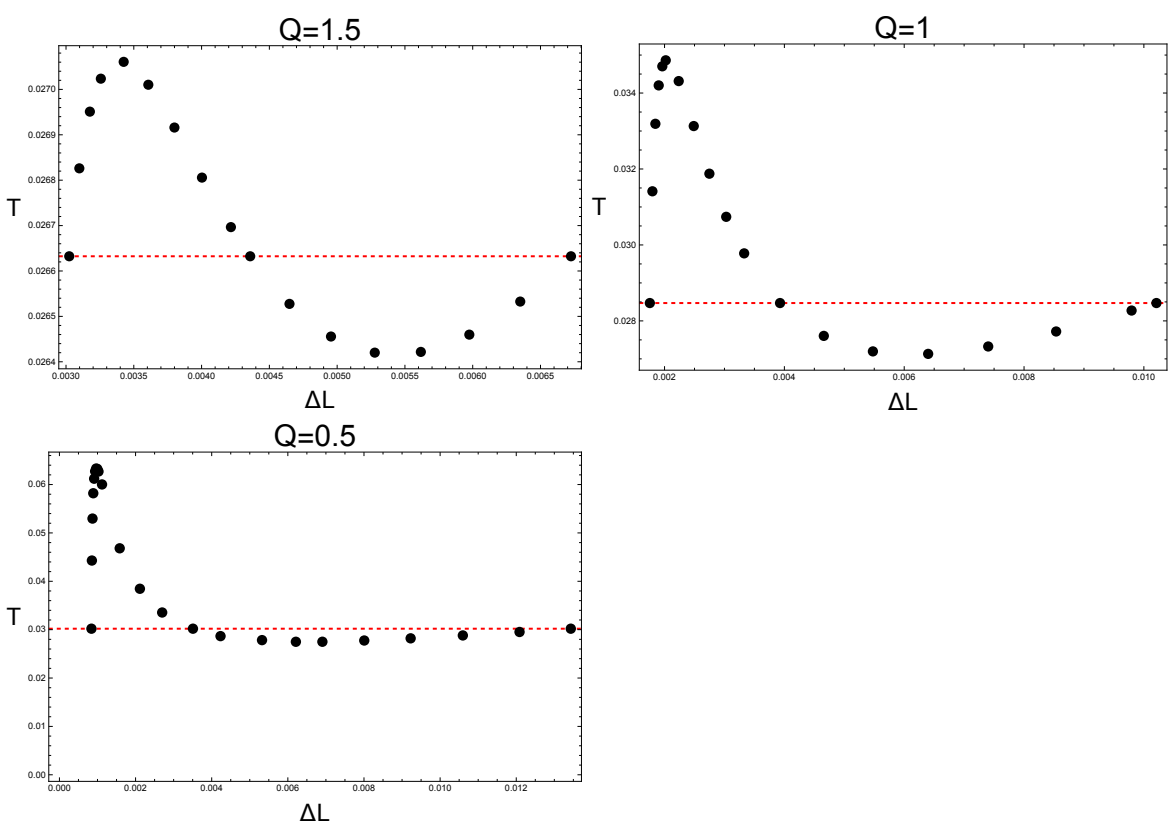

Figure 5. Charge dependence of $T-\Delta L$ diagrams. The plots are displayed for the $d=4$ charged ADS black holes. The phase transition temperature $T_{*}$ is plotted in red in each case. Again we have $l=10, \theta_{0}=0.15$, and $\theta_{c}=0.149$.

where $L\left(x_{i}, x_{j}\right)$ is the smallest bulk geodesic between $\left(t_{0}, x_{i}\right)$ and $\left(t_{0}, x_{j}\right)$. In order to formulate a Maxwell construction for the two-point correlation function, we can choose the points $x_{1}=\left(\theta=0, \phi=\frac{\pi}{2}\right), x_{2}=\left(\theta=\theta_{0}, \phi=\frac{\pi}{2}\right) . L\left(x_{1}, x_{2}\right)$ can be then computed by minimising the functional

$$
L\left(x_{1}, x_{2}\right)=\int_{0}^{\theta_{0}} \sqrt{\frac{r^{\prime}(\theta)^{2}}{f(r(\theta))}+r(\theta)^{2}} d \theta
$$

This is a similar computation to that carried out using (3.6). The quantity $L$ must be computed by solving the Euler-Lagrange equations, a cut off $\theta_{c}$ is chosen, and the vacuum AdS two point function $L_{0}$ is be subtracted off to obtain $\Delta L=L-L_{0}$. Again, the vacuum AdS solution is given by equation (3.7).

Contrary to claims [9-18] that when computed in this way $L$ obeys a Maxwell equal area construction, we find again that there is no equal area law in this plane as we move away from criticality. Our results are illustrated in figure 5 and table 2, where the relative error between Areas I and II is $45 \%$ at $Q=0.3 Q_{\text {crit }}$.

\section{Failure of the equal area construction}

Our results show that there is no numerical evidence for a holographic equal area law in either the black hole temperature/entanglement entropy or the black hole temperature/two point correlation function plane, at the phase transition temperature of the black hole. 


\begin{tabular}{|c|c|c|c|c|c|}
\hline$Q$ & $Q / Q_{c}$ & $T_{*}$ & Area(I) & Area(II) & Relative error \\
\hline 1.5 & 0.9 & 0.0266324 & $3.382 \times 10^{-7}$ & $3.313 \times 10^{-7}$ & $2.01 \%$ \\
\hline 1 & 0.6 & 0.02847 & $6.685 \times 10^{-6}$ & $5.350 \times 10^{-6}$ & $20.0 \%$ \\
\hline 0.5 & 0.3 & 0.030198 & $3.032 \times 10^{-5}$ & $1.667 \times 10^{-5}$ & $45.0 \%$ \\
\hline
\end{tabular}

Table 2. The failure of the equal area law for the two-point correlation function. Similarly as for $S_{E}$, as we move away from criticality we see a failure in the equal area law for $\Delta L$.

Similar evidence [7] for the failure of the equal area law for entanglement entropy has been attributed to the first law of entanglement (3.3), which we rewrite as [27]

$$
d E_{A}=T_{\mathrm{ent}} d S_{A},
$$

where $E_{A}$ is the energy contained in a region $A$ and $S_{A}$ is the entanglement entropy between a small region $A$ and its complement. We note that our restriction to only small values of $\theta_{0}$ ensures our calculations are well within the small-region regime. $T_{\text {ent }}$ is known as the entanglement temperature, defined by comparing the energy to entropy ratio $\frac{\Delta E_{A}}{\Delta S_{A}}$ for an excited state of the region A relative to the ground state for the same region in the CFT.

$E_{A}$ can be computed by integrating the stress tensor of the black hole spacetime on the boundary [28]:

$$
E_{A}=\int d^{d-2} x T_{t t}
$$

For spherically symmetric asymptotically AdS spacetime, upon computing the difference $\Delta E_{A} \equiv E_{A}-E_{A}^{(0)}$ between the excited and vacuum state in region $\mathrm{A}$ this becomes

$$
\Delta E_{A} \propto \int d^{d-2} x M,
$$

since $T_{t t}$ is proportional to the mass $M[29]$. For constant entangling region size we should therefore have

$$
T_{\text {ent }} S_{E} \propto M
$$

using (3.5); since $T_{\text {ent }}$ depends only on entangling region size [27], which is kept constant, we find

$$
S_{E} \propto M
$$

as a first law for the relative entanglement entropy. This relation is straightforwardly tested numerically. Indeed, when we plot $S_{E}$ against $M$ we find that these are proportional, as depicted in figure 6. From these graphs we see that while [27] only dealt with uncharged, asymptotically planar AdS spacetimes, its results are also valid in the charged asymptotically spherical case.

Thus, defining for an equal area law for entanglement entropy is equivalent to defining an equal area law in the $T / M$ plane. However, the Maxwell construction works in general only for pairs of conjugate thermodynamical variables: there is no equal area law in the $T / M$ plane. Thus, there is no reason to expect an equal area law in the $T / S_{E}$ plane. To study an equal area law for entanglement entropy, we would require a corresponding 
$Q=10 / 6=Q_{\text {crit }}$
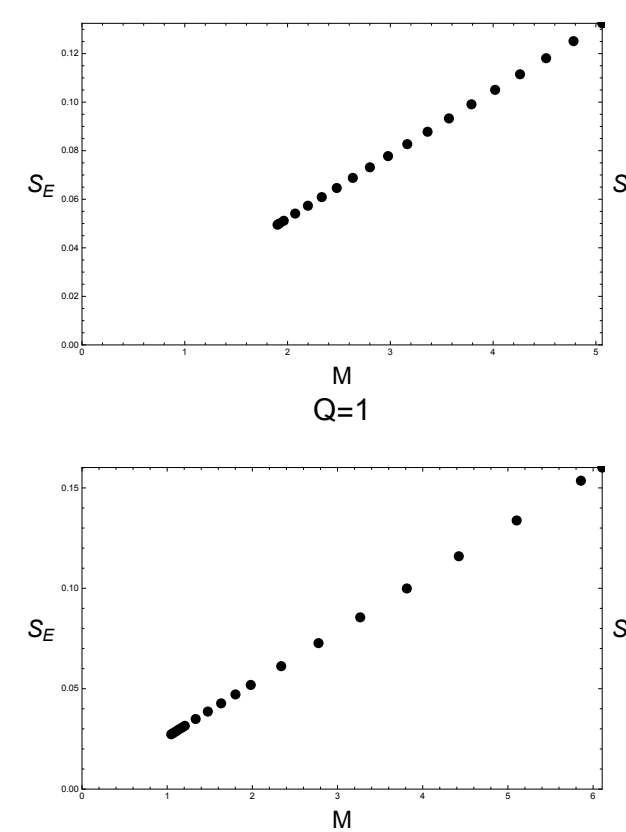

$\mathrm{Q}=1.5$
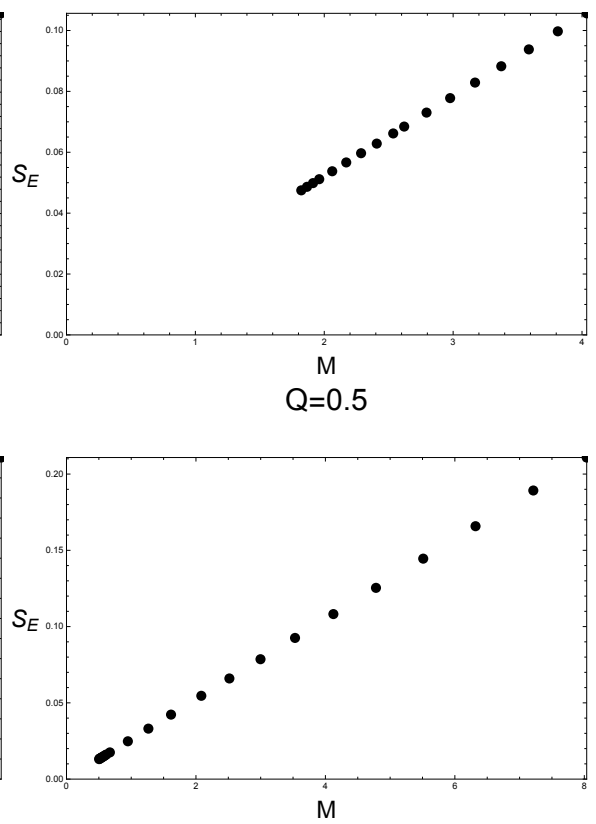

Figure 6. The first law for relative entanglement entropy. These plots show various isocharges in the black hole mass-entanglement entropy plane for the charged AdS black hole in $d=4$ dimensions. The graphs verify that $S_{E} \propto M$ and thus verify the first law of entanglement entropy.

\begin{tabular}{|c|c|c|}
\hline \multirow{2}{*}{$Q / Q_{c}$} & \multicolumn{2}{|c|}{ Relative Error } \\
\cline { 2 - 3 } & Areas: eq. (2.14) & Areas: eq. (2.11) \\
\hline 0.9 & $0.00389 \%$ & $1.12 \%$ \\
\hline 0.6 & $0.455 \%$ & $16.7 \%$ \\
\hline 0.3 & $3.17 \%$ & $42.1 \%$ \\
\hline
\end{tabular}

Table 3. Comparing the Accuracy of the errors given by Equations (2.14) and (2.11). We have computed the relative error on the $T / M$ plane between the areas defined by each equation. Due to the areas being defined by (2.14) being much larger, their relative error is less accurate than between the relative error between the areas of interest defined by $(2.11){ }^{2}$

thermodynamic interpretation of the free energy, and we would need to consider $S_{E}$ plotted against its thermodynamic conjugate. We likewise find that the two-point correlation function is proportional to $M$, as shown in figure 7 .

As noted previously, one of the main reasons for the discrepancy between our findings and those contending an equal area law has to do with using (2.14) instead of the more precise (2.11). In table 3 we illustrate this for several values of $Q / Q_{c}$. It is clear that the distinction can be very large, and it is clear that (2.11) provides no support for an equal area law.

\footnotetext{
${ }^{2}$ The discrepancies between the values given for the relative errors in the $T / M$ plane between tables 3 and 4 comes from the fact that the areas in table 3 were found exactly, whereas the areas in table 4 were
} 
$Q=1.5$
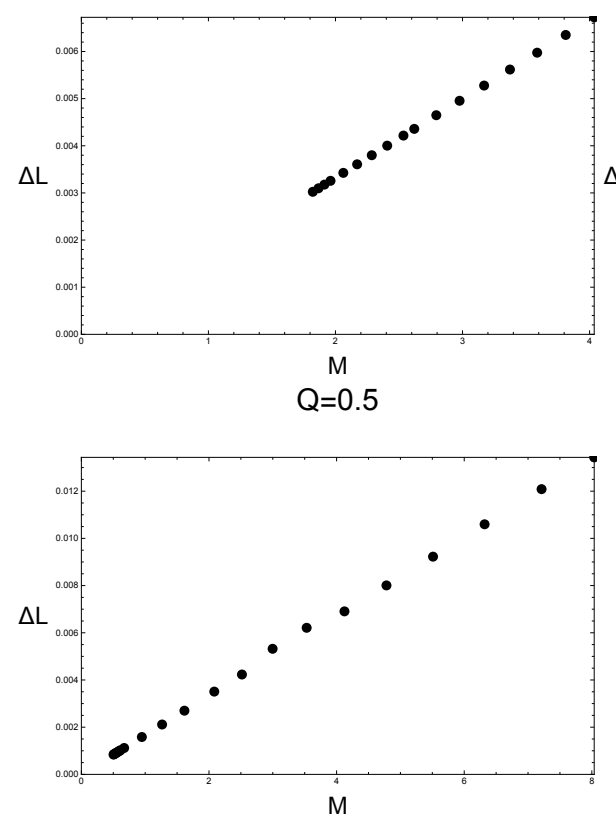

$Q=1$

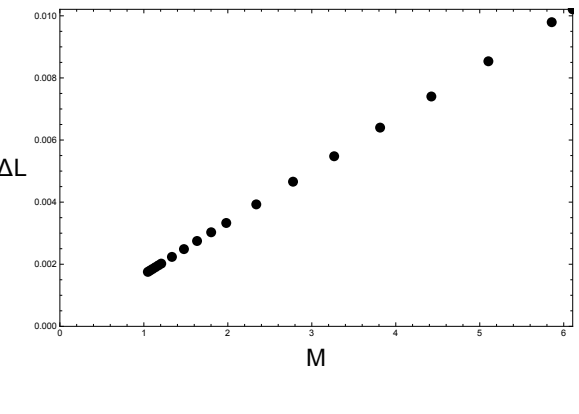

Figure 7. The proportionality of $\Delta L$ and $M$. These plots show the isocharges in the black hole mass- $\Delta L$ plane for the charged AdS black hole in $d=4$ dimensions. The graphs verify that $\Delta L \propto M$ and thus that there is not an equal area law for $\Delta L$.

\begin{tabular}{|c|c|c|c|c|}
\hline \multirow{2}{*}{$Q$} & \multirow{2}{*}{$Q / Q_{c}$} & \multicolumn{3}{|c|}{ Relative Error } \\
\cline { 3 - 5 } & & Mass & HEE & $\Delta L$ \\
\hline 1.5 & 0.9 & $1.96 \%$ & $2.02 \%$ & $2.01 \%$ \\
\hline 1 & 0.6 & $18.8 \%$ & $19.0 \%$ & $20.0 \%$ \\
\hline 0.5 & 0.3 & $44.2 \%$ & $44.1 \%$ & $45.0 \%$ \\
\hline
\end{tabular}

Table 4. Comparing the Relative Errors. We have computed the relative error between Areas I and II on the Temperature/Mass plane and we have compared this with those on the $T / \Delta S$ and $T / \Delta L$ planes. The numerical errors in both cases are very close to that in the $T / M$ plane.

We summarize in table 4 a comparison of the relative errors between Areas I and II on the $T / M, T / S_{E}$, and $T / \Delta L$ planes. In all cases we find that this quantity grows as the departure from criticality increases. We conclude that there no reason to expect an equal area law for either the two-point correlation function or for the entanglement entropy.

We close this section by commenting on the $\theta_{c}$ and $\theta_{0}$ dependence of our results in $S_{E}=S_{A}-S_{A}^{(0)}$. This manifests itself differently in $S_{A}$ and $S_{A}^{(0)}$ in such a way that the $\theta_{c}$ dependence of $S_{E}$ was numerically found to be given by the following expression

$$
S_{E}=\sin \theta_{0} \int_{0}^{\theta_{c}} \sin ^{d-3} x d x F\left(Q, r_{+}, l\right) .
$$

numerically integrated using only the masses at the points for which the $S_{E}$ and $\Delta L$ values were calculated, in order to obtain a more meaningful comparison. 
These relationships are not found in $S_{A}$ or in the background entanglement entropy $S_{A}^{(0)}$, but only in their difference $S_{E}$. We have numerically checked this for $d=4$ and 5 . Thus the proportionality between $S_{E}$ and $M$ is only dependent on either of these parameters via the proportionality constant: the slopes of the lines in Figs 6 and 7 will change, but the relative error between Areas I and II is uneffected.

\section{The "approximate" equal area law near criticality}

It is evident that much of the confusion in the literature on the subject of the holographic equal area law stems from the seemingly "approximate" equal area law obeyed on the $T / M$ plane near criticality. In 4 spacetime dimensions this can be explained by demonstrating that Areas I and II on the $T / M$ plane must approach zero at the same rate near criticality, which we can see by Taylor expanding expressions for these areas near criticality.

Namely, in 4 spacetime dimensions an expression for the phase transition temperature $T_{*}$, obtained by requiring that both the temperature and the free energy are equal for the large and small black holes, is exactly known $[4,30]$

$$
T_{*}=\frac{\sqrt{l-2 Q}}{l^{3 / 2} \pi} .
$$

Areas I and II are given by

$$
\begin{gathered}
\operatorname{Area}(\mathrm{I})=\int_{M_{1}}^{M_{2}} T d M-T_{*}\left(M_{2}-M_{1}\right), \\
\operatorname{Area}(\mathrm{II})=T_{*}\left(M_{3}-M_{2}\right)-\int_{M_{2}}^{M_{3}} T d M,
\end{gathered}
$$

where $M_{1}, M_{2}$, and $M_{3}$ are the masses $M\left(S_{i}, Q\right)$ corresponding to the three solutions $S_{1}, S_{2}$, $S_{3}$ of $T(S, Q)=T_{*}$. We note that the Areas (I) and (II) as defined above approaching zero at the same rate is equivalent to the larger areas Area $(\mathrm{A})=T_{*}\left(M_{3}-M_{1}\right)$ and Area $(\mathrm{B})=$ $\int_{M_{1}}^{M_{3}} T d M$ approaching zero at the same rate and so it suffices to look at the near-critical expansions of areas A and B. $S_{1}$ and $S_{3}$ are known [4, 30]:

$$
S_{1}=\frac{4 l^{2} \pi Q^{2}}{(\sqrt{l(l-6 Q)}+\sqrt{l(l-2 Q)})^{2}}, \quad S_{3}=\frac{\pi}{4}(\sqrt{l(l-6 Q)}+\sqrt{l(l-2 Q)})^{2} .
$$

This allows us to find expressions for $M_{1}$ and $M_{3}$ from $M=\frac{S^{2}+l^{2} \pi\left(\pi Q^{2}+S\right)}{2 l^{2} \pi^{3 / 2} \sqrt{S}}$. After doing this, we can expand areas A and B about the critical charge $Q_{c r i t}=l / 6$, with $\delta q=\left(Q_{c}-Q\right) / l$ :

$$
\begin{aligned}
\operatorname{Area}(\mathrm{A}) & =T_{*}\left(M_{3}-M_{1}\right) \\
& =\frac{4}{3 \pi}(\delta q)^{1 / 2}+\frac{6}{\pi}(\delta q)^{3 / 2}+\frac{9}{2 \pi}(\delta q)^{5 / 2}-\frac{9}{4 \pi}(\delta q)^{7 / 2}+\ldots \\
\text { Area }(\mathrm{B}) & =\int_{M_{1}}^{M_{3}} T(S, Q) d M=\int_{S_{1}}^{S_{3}} T \frac{\partial M}{\partial S} d S \\
& =\frac{4}{3 \pi}(\delta q)^{1 / 2}+\frac{6}{\pi}(\delta q)^{3 / 2}+\frac{9}{2 \pi}(\delta q)^{5 / 2}+\frac{2277}{140 \pi}(\delta q)^{7 / 2}+\ldots,
\end{aligned}
$$




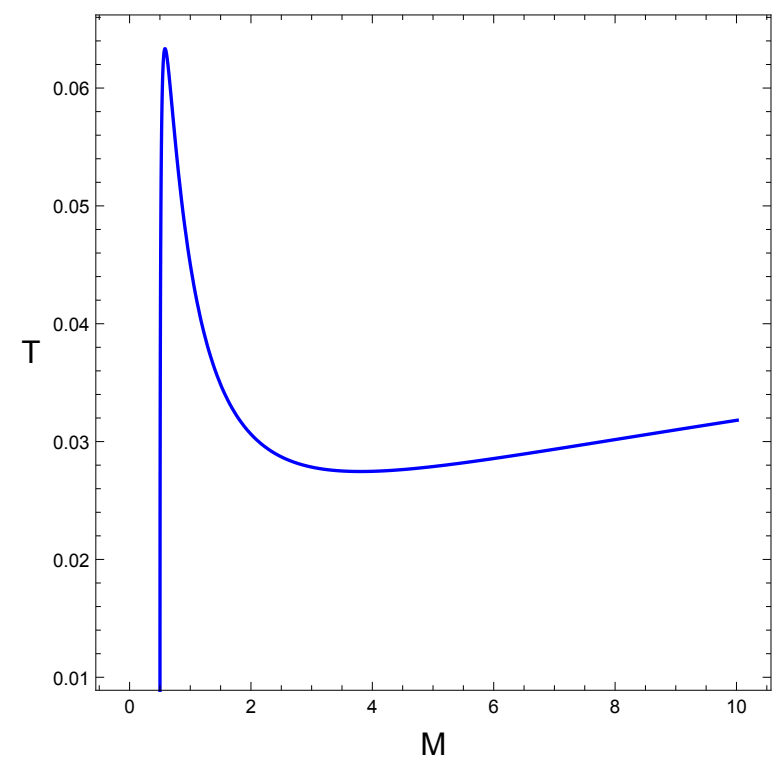

Figure 8. $T$ versus $M . T$ is plotted against $M$ in $d=4$, with $l=10$ and $Q=0.5=0.3 Q_{c}$. We see the oscillatory behaviour of $T$; our $T / S_{E}$ and $T / \Delta L$ graphs (Figs 4 and 5 ) are just rescaled versions of this plot.

from which we can see that Areas A and B agree up to the first three terms of the Taylor expansion. This explains the appearance of an equal law near criticality when $\delta q$ is small. A slightly more general argument, valid for other black holes and in any dimension, is presented in appendix A.

\section{Conclusions}

The observation [23] that relative entanglement entropy displays qualitatively similar behaviour to black hole entropy on isocharges of the $3+1$-dimensional charged AdS black hole below criticality seemed intriguing. Nguyen attempted to sharpen this similarity in [5] by showing that the relative entanglement entropy obeys an equal area construction, evidence for a phase transition. Further claims that a holographic equal area law holds in the $T /$ geodesic length plane have also been put forward (see references above).

Our results indicate, commensurate with [7], that all proposals thus far put forward that a form of Maxwell's equal area law holds for entanglement entropy are false (although it is 'almost satisfied' near criticality, see table 4). Moreover, we do not find it surprising that the isocharges display oscillatory behaviour below $Q_{c r i t}$ and a point of inflection at $Q_{\text {crit }}$ on the $T-S_{E}$ plane. This is a simple consequence of two facts: i) $S_{E}=S_{E}(M)$ is a monotonic function due to the first law for the relative entanglement entropy and ii) temperature $T$, when displayed as a function of $M$, demonstrates oscillatory behavior, as shown in figure 8 , with the oscillation disappearing at criticality.

After numerically studying the holographic Maxwell construction for entanglement entropy and the two-point correlation function, we find no reason to support such proposals for the equal area law. The entanglement entropy is not dual to the black hole entropy, 
and it should not be expected that it obeys an equal area law. Any equal area construction for entanglement entropy should be studied in relation to its thermodynamic dual, and any claim of a phase transition must be backed up by a free energy diagram on the boundary similar to that in figure 1; in other words there must be an analogue of free energy that displays swallowtail behavior.

While we expect that phase transition for a bulk black hole has a counterpart in the boundary CFT, although the entanglement entropy jumps in such a phase transition, the transition temperature is not given by the associated equal area law. A CFT phase transition will be governed by the corresponding free energy of CFT. Translating this into a holographic equal area law of some kind remains an open question.

\section{Acknowledgments}

We would like to thank the anonymous referee for helping us to improve our manuscript. This research was supported in part by Perimeter Institute for Theoretical Physics and by the Natural Sciences and Engineering Research Council of Canada. Research at Perimeter Institute is supported by the Government of Canada through the Department of Innovation, Science and Economic Development Canada and by the Province of Ontario through the Ministry of Research, Innovation and Science.

\section{A The approximate equal area law: general argument}

In section 5 we presented an argument as to why there appears to be an "approximate" equal area law near criticality in the $T / M$ plane for charged AdS black holes in 4 dimensions. Here we demonstrate that this holds more generally, in any number of dimensions and for other black hole solutions for which the expressions for $T_{*}$, and $M_{1}$ and $M_{3}$ are not explicitly known.

Areas $\mathrm{A}$ and $\mathrm{B}$ are given by

$$
\begin{aligned}
& \operatorname{Area}(\mathrm{A})=T_{*}\left(M_{3}-M_{1}\right), \\
& \operatorname{Area}(\mathrm{B})=\int_{M_{1}}^{M_{3}} T d M .
\end{aligned}
$$

Assuming that for a chosen black hole there is an equal area law satisfied in the $T / S$ plane, we can find an expression for $T_{*}$ :

$$
T_{*}=\frac{\int_{S_{1}}^{S_{3}} T d S}{S_{3}-S_{1}},
$$

such that

$$
\operatorname{Area}(\mathrm{A})=\frac{M_{3}-M_{1}}{S_{3}-S_{1}} \int_{S_{1}}^{S_{3}} T d S .
$$

To calculate the integrals (A.2) and (A.4) we expand

$$
\begin{aligned}
T=T(S, Q) & =T_{c}+\frac{\partial T}{\partial S} \Delta S+\frac{\partial T}{\partial Q} \Delta Q+\ldots \\
& =T_{c}+\frac{\partial T}{\partial Q} \Delta Q+\ldots,
\end{aligned}
$$


where $\Delta Q \equiv Q-Q_{c}, \Delta S=S-S_{c}$, and it is understood that the derivatives are evaluated at $Q_{c}, S_{c}$; the last equality follows from the fact that at criticality $\partial T / \partial S=0$. So we have

$$
\begin{aligned}
\operatorname{Area}(\mathrm{A}) & =\frac{M_{3}-M_{1}}{S_{3}-S_{1}} \int_{S_{1}}^{S_{3}}\left(T_{c}+\frac{\partial T}{\partial Q} \Delta Q\right) d S \\
& =\left(T_{c}+\frac{\partial T}{\partial Q} \Delta Q\right)\left(M_{3}-M_{1}\right) .
\end{aligned}
$$

At the same time we have

$$
\begin{aligned}
\operatorname{Area}(\mathrm{B}) & =\int_{M_{1}}^{M_{3}} T d M=\int_{M_{1}}^{M_{3}}\left(T_{c}+\frac{\partial T}{\partial Q} \Delta Q\right) d M \\
& =\left(T_{c}+\frac{\partial T}{\partial Q} \Delta Q\right)\left(M_{3}-M_{1}\right)
\end{aligned}
$$

and hence the areas are equal to this order of expansion in $\Delta Q$. Since $\left(M_{3}-M_{1}\right)$ has to go to zero near criticality, the above formulas show that the areas are equal at least to the order linear in $\Delta Q$.

Open Access. This article is distributed under the terms of the Creative Commons Attribution License (CC-BY 4.0), which permits any use, distribution and reproduction in any medium, provided the original author(s) and source are credited.

\section{References}

[1] E. Spallucci and A. Smailagic, Maxwell's equal area law for charged Anti-deSitter black holes, Phys. Lett. B 723 (2013) 436 [arXiv:1305.3379] [INSPIRE].

[2] S.-Q. Lan, J.-X. Mo and W.-B. Liu, A note on Maxwell's equal area law for black hole phase transition, Eur. Phys. J. C 75 (2015) 419 [arXiv:1503.07658] [INSPIRE].

[3] H. Xu and Z.-M. Xu, Maxwell's equal area law for Lovelock thermodynamics, Int. J. Mod. Phys. D 26 (2016) 1750037 [arXiv: 1510.06557] [INSPIRE].

[4] D. Kubiznak, R.B. Mann and M. Teo, Black hole chemistry: thermodynamics with Lambda, Class. Quant. Grav. 34 (2017) 063001 [arXiv: 1608.06147] [INSPIRE].

[5] P.H. Nguyen, An equal area law for holographic entanglement entropy of the AdS-RN black hole, JHEP 12 (2015) 139 [arXiv: 1508.01955] [INSPIRE].

[6] X.-X. Zeng, H. Zhang and L.-F. Li, Phase transition of holographic entanglement entropy in massive gravity, Phys. Lett. B 756 (2016) 170 [arXiv:1511.00383] [INSPIRE].

[7] Y. Sun, H. Xu and L. Zhao, Thermodynamics and holographic entanglement entropy for spherical black holes in 5D Gauss-Bonnet gravity, JHEP 09 (2016) 060 [arXiv:1606.06531] [INSPIRE].

[8] X.-M. Liu, H.-B. Shao and X.-X. Zeng, Van der Waals-like phase transition from holographic entanglement entropy in Lorentz breaking massive gravity, Adv. High Energy Phys. 2017 (2017) 6402101 [arXiv:1706.04431] [InSPIRE].

[9] X.-X. Zeng and L.-F. Li, Van der Waals phase transition in the framework of holography, Phys. Lett. B 764 (2017) 100 [arXiv:1512.08855] [inSPIRE]. 
[10] X.-X. Zeng, X.-M. Liu and L.-F. Li, Phase structure of the Born-Infeld-anti-de Sitter black holes probed by non-local observables, Eur. Phys. J. C 76 (2016) 616 [arXiv:1601.01160] [INSPIRE].

[11] J.-X. Mo, G.-Q. Li, Z.-T. Lin and X.-X. Zeng, Revisiting van der Waals like behavior of $f(R)$ AdS black holes via the two point correlation function, Nucl. Phys. B 918 (2017) 11 [arXiv: 1604.08332] [INSPIRE].

[12] J.-X. Mo, An alternative perspective to observe the critical phenomena of dilaton black holes, Eur. Phys. J. C 77 (2017) 529 [arXiv: 1607.03702] [InSPIRE].

[13] S. He, L.-F. Li and X.-X. Zeng, Holographic Van der Waals-like phase transition in the Gauss-Bonnet gravity, Nucl. Phys. B 915 (2017) 243 [arXiv: 1608.04208] [inSPIRE].

[14] X.-X. Zeng and L.-F. Li, Holographic Phase Transition Probed by Nonlocal Observables, Adv. High Energy Phys. 2016 (2016) 6153435 [arXiv: 1609.06535] [INSPIRE].

[15] H. El Moumni, Phase Transition of AdS Black Holes with Non Linear Source in the Holographic Framework, Int. J. Theor. Phys. 56 (2017) 554 [INSPIRE].

[16] H.-L. Li, S.-Z. Yang and X.-T. Zu, Holographic research on phase transitions for a five dimensional AdS black hole with conformally coupled scalar hair, Phys. Lett. B 764 (2017) 310 [INSPIRE].

[17] H.-L. Li and Z.-W. Feng, Holographic Van der Waals phase transition of the higher dimensional electrically charged hairy black hole, arXiv:1706.05530 [INSPIRE].

[18] X.-X. Zeng and Y.-W. Han, Holographic Van der Waals phase transition for a hairy black hole, Adv. High Energy Phys. 2017 (2017) 2356174 [arXiv:1706.02024] [INSPIRE].

[19] A. Chamblin, R. Emparan, C.V. Johnson and R.C. Myers, Charged AdS black holes and catastrophic holography, Phys. Rev. D 60 (1999) 064018 [hep-th/9902170] [InSPIRE].

[20] A. Chamblin, R. Emparan, C.V. Johnson and R.C. Myers, Holography, thermodynamics and fluctuations of charged AdS black holes, Phys. Rev. D 60 (1999) 104026 [hep-th/9904197] [INSPIRE].

[21] D. Kubiznak and R.B. Mann, P-V criticality of charged AdS black holes, JHEP 07 (2012) 033 [arXiv: 1205.0559] [INSPIRE].

[22] V. Balasubramanian and S.F. Ross, Holographic particle detection, Phys. Rev. D 61 (2000) 044007 [hep-th/9906226] [inSPIRE].

[23] C.V. Johnson, Large-N Phase Transitions, Finite Volume and Entanglement Entropy, JHEP 03 (2014) 047 [arXiv: 1306.4955] [INSPIRE].

[24] D.D. Blanco, H. Casini, L.-Y. Hung and R.C. Myers, Relative Entropy and Holography, JHEP 08 (2013) 060 [arXiv: 1305.3182] [INSPIRE].

[25] G. Wong, I. Klich, L.A. Pando Zayas and D. Vaman, Entanglement Temperature and Entanglement Entropy of Excited States, JHEP 12 (2013) 020 [arXiv:1305.3291] [INSPIRE].

[26] S. Ryu and T. Takayanagi, Holographic derivation of entanglement entropy from AdS/CFT, Phys. Rev. Lett. 96 (2006) 181602 [hep-th/0603001] [INSPIRE].

[27] J. Bhattacharya, M. Nozaki, T. Takayanagi and T. Ugajin, Thermodynamical Property of Entanglement Entropy for Excited States, Phys. Rev. Lett. 110 (2013) 091602 [arXiv: 1212.1164] [INSPIRE]. 
[28] J.D. Brown and J.W. York Jr., Quasilocal energy and conserved charges derived from the gravitational action, Phys. Rev. D 47 (1993) 1407 [gr-qc/9209012] [INSPIRE].

[29] V. Balasubramanian and P. Kraus, A Stress tensor for Anti-de Sitter gravity, Commun. Math. Phys. 208 (1999) 413 [hep-th/9902121] [InSPIRE].

[30] J.-X. Mo and G.-Q. Li, Coexistence curves and molecule number densities of AdS black holes in the reduced parameter space, Phys. Rev. D 92 (2015) 024055 [arXiv:1604.07931] [INSPIRE]. 